\title{
Transtorno do Espectro Autista e as tecnologias educacionais digitais no cenário das pesquisas brasileiras: um Mapeamento Sistemático da Literatura
}

\author{
Autistic Spectrum Disorder and digital educational technologies in the Brazilian research scenario:
} a Systematic Literature Mapping

Trastorno del espectro autista y tecnologías educativas digitales en el escenario de investigación brasileño: un mapeo sistemático de la literatura

Recebido: 16/07/2021 | Revisado: 29/07/2021 | Aceito: 01/08/2021 | Publicado: 06/08/2021

\author{
Dario Silva da Silva Júnior \\ ORCID: https://orcid.org/0000-00002-6588-4455 \\ Universidade Federal do Tocantins, Brasil \\ E-mail: dario.junior@mail.uft.edu.br \\ Patrick Letouzé Moreira \\ ORCID: https://orcid.org/0000-00001-7728-3254 \\ Universidade Federal do Tocantins, Brasil \\ E-mail: letouze@mail.uft.edu.br
}

\begin{abstract}
Resumo
O Transtorno do Espectro Autista (TEA) é uma alteração neurocomportamental de diagnóstico médico e tratamento interdisciplinar, que envolve um relacionamento translacional das diversas áreas da saúde, visando a minimização do quadro clínico do paciente. Observa-se que o processo de aprendizagem das crianças e adolescentes com o TEA é mais dificultoso em relação a seus pares sem o transtorno. Este artigo contempla um mapeamento sistemático da literatura, utilizando uma modificação do protocolo PICO, sobre as tecnologias educacionais e tecnologias da informação estudadas para melhorar o processo de ensino-aprendizagem de crianças com o TEA. O mapeamento considerou artigos das bases de dados disponíveis no Google Acadêmico. Observa-se nos trabalhos analisados que a utilização de tecnologias de informação e comunicação tiveram resultados promissores no processo da aprendizagem das crianças do TEA. Contudo, percebe-se que para se tornar algo factível, é necessário um adequado planejamento pedagógico, acurácia no diagnóstico das necessidades individuais de cada criança frente a sua deficiência e melhor manejo dos professores com as tecnologias do mercado.
\end{abstract}

Palavras-chave: Educação especial; Métodos de ensino; Mobilidade estudantil; Rendimento; Transversalidade.

\begin{abstract}
The Autistic Spectrum Disorder (ASD) is a neurobehavioral alteration of medical diagnosis and interdisciplinary treatment, which involves a translational relationship of different areas of health, aiming at minimizing the patient's clinical condition. It is observed that the learning process of children and adolescents with ASD is more difficult compared to their peers without the disorder. This article contemplates a systematic literature mapping, using a modification of the PICO protocol, about the educational technologies and information technologies studied to improve the teaching-learning process of children with ASD. The mapping considered articles from the databases available on Google Scholar. It is observed in the analyzed works that the use of information and communication technologies had promising results in the learning process of children in the TEA. However, it is clear that to become something feasible, it is necessary to have an adequate pedagogical planning, accuracy in diagnosing the individual needs of each child facing their disability and better management of teachers with market Technologies.
\end{abstract}

Keywords: Special education; Teaching methods; Student mobility; Performance; Transversality.

\section{Resumen}

El Trastorno del Espectro Autista (TEA) es un trastorno neuroconductual de diagnóstico médico y tratamiento interdisciplinario, que implica una relación traslacional de diferentes áreas de la salud, con el objetivo de minimizar la condición clínica del paciente. Se observa que el proceso de aprendizaje de los niños y adolescentes con TEA es más difícil en comparación con sus compañeros sin el trastorno. Este artículo contempla un mapeo sistemático de la literatura, a través de una modificación del protocolo PICO, sobre las tecnologías educativas y de la información estudiadas para mejorar el proceso de enseñanza-aprendizaje de niños con TEA. El mapeo consideró artículos de las bases de datos disponibles en Google Scholar. Se observa en los trabajos analizados que el uso de las tecnologías de la información y la comunicación tuvo resultados prometedores en el proceso de aprendizaje de los niños en TEA. Sin embargo, es claro que para convertirse en algo viable, es necesario contar con una adecuada planificación pedagógica, 
precisión en el diagnóstico de las necesidades individuales de cada niño frente a su discapacidad y una mejor gestión de los docentes con tecnologías de mercado.

Palabras clave: Educación especial; Métodos de enseñanza; Movilidad estudiantil; Rendimiento; Transversalidad.

\section{Introdução}

O Transtorno do Espectro Autista - doravante denominado de TEA, era antigamente denominado Autismo. O TEA consiste numa síndrome neurocomportamental complexa que afeta a linguagem e o desenvolvimento cognitivo e social, presença de comportamentos repetitivos, além de interesses ou atividades restritas (Mukherjee et al., 2020; American Psychiatric Association, 2014). Esta nomenclatura é mais aceita pela comunidade médica e científica, uma vez que, existe uma variedade grande de sinais e sintomas nesse transtorno (De Bettio \& Giacomazzo, 2020). O TEA pode ser definido como uma perda significativa nas interações sociais, caracterizada por um dos seguintes itens: problemas com o uso de comportamento não-verbal; pouco contato visual; dificuldade de desenvolver relações com colegas; falta de vontade de compartilhar interesses e prazeres com terceiros; falta de reciprocidade emocional e social (American Psychiatric Association, 2014).

O desenvolvimento prejudicado da interação social e comunicação, com prejuízos em determinadas atitudes, como comportamento não verbal (contato visual, gestos corporais, expressão facial) comprometem e limitam o aprendizado (Teixeira, Santos, Aquino \& Medeiros, 2017). O modelo tradicional de educação é baseado na comunicação verbal e na relação interpessoal do professor e aprendente. Tal método dificulta o desenvolvimento do aprendizado de crianças com TEA frente às características próprias da síndrome, como os déficits de comunicação e interação social. Desde o século passado são estudadas diferentes formas de auxiliar e apoiar o desenvolvimento nessas crianças (Andreis \& Rigo, 2018).

Os processos de ensino e aprendizado já não são mais os mesmos, ou seja, estáticos. As demandas sociais estão cada vez mais necessitando de um ensino de qualidade, exigindo que crianças com o TEA recebam uma educação transformadora (Maia \& Jacomelli, 2020). Portanto, diversos estudos se engajam em estudar o processo de ensino-aprendizagem em crianças e/ou adolescentes com TEA com intuito de melhorar o rendimento, à medida que seus déficits são minimizados com auxílio da tecnologia vigente (De Bettio \& Giacomazzo, 2020; Maia \& Jacomelli, 2020; Nascimento, Luna, Espquincalha \& dos Santos, 2020; Silveira, Luiz, Guiterres, Mendes \& Ribeiro, 2020; Lucian \& Stumpf, 2019; Andreis \& Rigo, 2018; Kanashiro \& Seabra Júnior, 2018; Oliveira, Guebert \& Nohana, 2018; Pontes et al., 2018; Andrade \& Teodoro, 2017; Brito \& Nôvoa, 2017; Soares et al., 2017; Teixeira et al., 2017; Passerino, Bez \& Vicari, 2013).

A Constituição Federal do Brasil, em seu artigo 208 e a Lei número 12.764 de 27 de dezembro de 2012 preconizam que sejam incluídas nas escolas regulares as crianças e adolescentes com TEA, assim como, incentivam a capacitação de professores para atuar de forma adequada nessa inclusão. Contudo, a inclusão desses alunos na escola é difícil, tendo sido experimentadas e estudadas as tecnologias assistivas (TA) e as tecnologias da informação e comunicação (TIC's) como alternativas no processo de aprendizagem dessas crianças diagnosticas com o TEA (Andreis \& Rigo, 2018).

Atualmente as crianças crescem em uma sociedade tecnológica, principalmente a audiovisual e a digital, e desenvolvem grande aptidão para captar suas mensagens, mas nem sempre essa tecnologia é utilizada pelas escolas para melhorar o aprendizado (Maia \& Jacomelli, 2020); os avanços nas TIC's podem contribuir para ampliar as abordagens de educadores, profissionais de saúde e outros envolvidos no desenvolvimento integral da criança com o TEA (Pontes et al. 2018). Para De Bettio e Giacomazzo (2020) as TA trabalham a comunicação verbal, ortográfica, escrita, interpretação de texto, noção matemática e raciocínio lógico, o que aprimora, dessa maneira, o conhecimento e aprendizagem de cada aluno. Por conseguinte, o objetivo deste mapeamento sistemático da literatura é apresentar o cenário das pesquisas brasileiras sobre as tecnologias educacionais usadas no processo de ensino-aprendizagem em crianças e adolescentes com TEA. 


\section{Metodologia}

Para esta pesquisa foi realizado um mapeamento sistemático da literatura sobre as tecnologias educacionais utilizadas para o ensino-aprendizagem em crianças e adolescentes com TEA. O mapeamento sistemático é composto de um planejamento de pesquisa, formulação das questões a serem pesquisadas, definição dos critérios de inclusão e exclusão, além da busca dos artigos e a revisão dos trabalhos selecionáveis (Moreira, Souza Júnior \& Silva, 2016). As questões deste estudo foram desenvolvidas a partir de uma variação do Protocolo PICO (Population, Intervention, Comparison, Outcomes), inicialmente publicado por Richardson, Wilson, Nishikawa \& Hayward (1995) que sustenta a medicina baseada em evidência, contudo muito bem aplicável em outras áreas do conhecimento. Nessa estratégia foi utilizado o anagrama PIO (Population - população, Intervention - intervenção, Outcomes - resultados), frente a não necessidade de comparação de intervenções analisadas, conforme a Tabela 1 .

Tabela 1. Estratégia de investigação utilizada no Estudo.

\begin{tabular}{cc}
\hline $\begin{array}{c}\text { P }- \text { Population } \\
\text { População }\end{array}$ & Crianças e adolescentes com TEA. \\
$\begin{array}{c}\text { I }- \text { Intervention } \\
\text { Intervenção }\end{array}$ & $\begin{array}{c}\text { Aplicação, comparação ou revisão da literatura sobre Tecnologias de } \\
\text { informação e comunicação, realidade aumentada, tecnologia assistiva } \\
\text { no processo de ensino-aprendizagem. }\end{array}$ \\
\hline $\begin{array}{c}\text { O - Outcomes } \\
\text { Resultados }\end{array}$ & Impactos no processo de ensino e aprendizagem. \\
\hline
\end{tabular}

Fonte: Autores.

Conforme evidenciado, o item Population envolvem crianças e adolescentes com TEA. O item Intervention representa as ferramentas e os meios que ajudam a solucionar o questionamento de cada trabalho e Outcomes é o desfecho do questionamento. Dessa forma a questão do presente estudo é: crianças e adolescentes com TEA poderiam se favorecer com uso de tecnologia educacionais no processo de ensino-aprendizagem?

Para isso, utilizou-se como ferramenta de busca o Google Acadêmico, com os buscadores "transtorno do espectro autista" "tecnologia educacional" "tecnologias educacionais" "tecnologia da informação". A seguinte equação de busca foi utilizada na modalidade pesquisa avançada: "transtorno do espectro autista" AND "tecnologia educacional" AND "tecnologias educacionais" AND "tecnologia da informação". A pesquisa foi realizada nos dias 17 e 18 de novembro de 2020 . Os documentos encontrados foram transferidos para Biblioteca do Google Acadêmico e posteriormente armazenados no programa Zotero® para posterior análise, realizada entre os dias 19 e 30 de novembro, respeitando como critérios de inclusão: assuntos relacionados com educação e tecnologias educacionais no TEA; idiomas em português, inglês e espanhol; periódico de revista científica da área da saúde ou educação ou informática; artigos completos. Os critérios de exclusão aplicados: outra língua que não português, inglês ou espanhol; outra forma de divulgação que não um artigo de periódico científico de saúde ou educação ou informática; TEA relacionado com outros temas que não tecnologias educacionais e artigos incompletos ou repetidos. Este trabalho não foi submetido à apreciação do Comitê de Ética em Pesquisa por se tratar de uma revisão de literatura.

\section{Resultados}

A pesquisa demonstrou, aproximadamente, 714 documentos, porém com contagem exata de 698 documentos passíveis de extração para análise. Foi realizada a leitura do título e do resumo de cada artigo, sendo que 30 documentos foram selecionados; porém quatro, dentre os artigos selecionados, foram excluídos respeitando os critérios de inclusão e exclusão, tendo sido separados 26 artigos para análise na íntegra. A análise pormenorizada ocorreu com a leitura integral de cada artigo visando a busca elementos guiados pela estratégia PICO modificada descrita na metodologia. Dessa forma, restou ao final da 
seleção apenas 14 artigos que se relacionam totalmente com o escopo desta pesquisa. Segue abaixo os trabalhos analisados conforme metodologia do protocolo PICO modificado:

- Andrade e Teodoro (2017) desenvolveram um aplicativo de comunicação de metas entre cuidadores e profissionais da saúde no tratamento de pessoas com TEA (Population); Aplicaram e validaram o protótipo desenvolvido (Educautism®), um software personalizado para pais, tutores, profissionais da saúde auxiliando no processo de aprendizagem por jogos educativos, animações entre outros (Intervention); O sistema proposto pode auxiliar na resolução do problema da educação em crianças com TEA (Outcome).

- Andreis e Rigo (2018) apresentaram um sistema que auxilia na resolução de uso e adaptação à software (Educautism $\left.{ }^{\circledR}\right)$ de apoio à educação de crianças com TEA utilizando um Smartfhone com Sistema Android (Population); o sistema oferece nova proposta de interação ao usuário, com interface personalizada, de acordo com o perfil da criança com TEA. (Intervention); O modelo de interação criado através de uma interface personalizável evita crises nervosas e repúdio dos usuários às atividades, contribuindo com a qualidade de vida dos envolvidos no processo (Outcome).

- De Bettio e Giacomazzo (2020) buscaram identificar a presença da tecnologia assistiva no processo de aprendizagem dos alunos com TEA (Population); através de levantamento bibliográfico (Intervention), os autores demonstraram que os alunos conseguiram desenvolver a oralidade, comunicação e percepção numérica, ampliação da linguagem e motricidade, melhorando a competência matemática a capacidade funcional (Outcome).

- Kanashiro e Seabra Júnior (2018) investigaram o uso de tecnologia assistiva, denominada Jornada nas Estrelas (Intervention) em uma aluna com TEA no ensino fundamental (Population); observaram que o jogo não é capaz de alfabetizar, sendo necessário uma estratégia de intervenção do professor, portanto uma mediação para que se obtenha o êxito no processo de ensino e aprendizagem (Outcome).

- Lucian e Stumpf (2019) realizaram estudo de análise comparativa entre aplicativos educacionais para crianças com TEA (Population); analisaram os aplicativo ABC Autismo, TEO Autismo, Autismo Projeto Integrar. Após análises preliminares escolheu-se o ABC Autismo para a realização dos testes de viabilidade em crianças com TEA (Intervention); foi observado maior interação dos participantes com o uso do aplicativo, contudo algumas dificuldades foram salientadas como falta de comando por voz para o reconhecimento de letras e números (Outcome).

- Maia e Jacomelli (2020) analisaram a aprendizagem de criança com o TEA (Population) através de pesquisa bibliográfica, tendo como objetivo geral identificar se as Tecnologias de Informação e Comunicação (TIC) (Intervention); A utilização das TIC, tanto TV e vídeo quanto internet (desenhos animados), jogos em tablet e computadores contribuem para processo de ensino-aprendizagem, desde que seja realizado com a mentoria de um educador ou professor extraindo a pedagogia envolvida em cada TIC (Outcome).

- Nascimento et al. (2020) através de levantamento bibliográfico no ensino da matemática ligado ao pensamento numérico, às noções de número, contagem, classificação, seriação, quantidade, sequenciação, equivalência, ordem, e às quatro operações matemáticas envolvendo números com um ou dois algarismos (Intervention) em crianças com TEA através Tecnologias digitais (Population); Como resultado, houve aumento do interesse dos alunos pelos conteúdos e atividades frente às tecnologias digitais como jogos no computador ou aplicativos (Scratch®) (Outcome).

- Brito e Novôa (2017) buscando a inclusão de crianças com TEA (Population) relataram experiências de docentes com tecnologias digitais como o Amplisoft ${ }^{\circledR}$, AIELLO®, Descobrindo Emoções ${ }^{\circledR}$, Lina Educa ${ }^{\circledR}$, Scala ${ }^{\circledR}$, Emoplay ${ }^{\circledR}$ e os aplicativos CAN Game Maker® e ABC Autismo® e tecnologias não digitais como painel de rotina (Intervention); os autores concluem que não se necessita de tecnologias sofisticadas para um ensino de qualidade. As ferramentas devem ser funcionais e específicas às características de cada indivíduo, suprindo necessidades da escolarização e interação para desenvolverem autonomia (Outcome). 
- Oliveira et al. (2018) desenvolveram um sistema WEB que apresenta de modo digital as atividades de ensinoaprendizagem pela metodologia TEACCH para crianças com TEA (Population); o computador atua como um sistema WEB de apoio às atividades do método TEACCH para auxílio ao aprendizado de crianças com TEA (Intervention); O sistema portou-se de forma eficiente. Produziu melhoras nos resultados das avaliações que, no início da intervenção, mostraram-se insuficientes para o desenvolvimento cognitivo das crianças com TEA (Outcome).

- Passerino et al. (2013) realizaram um recorte do projeto SCALA (Sistema de comunicação alternativa para letramento de crianças com autismo) (Population); Discute-se a comunicação alternativa no espaço educativo para crianças com TEA, abordando a formação específica para os professores (Intervention); O projeto SCALA mostra-se efetivo como recurso do desenvolvimento de crianças com TEA, sendo importante a capacitação do professor para o uso das tecnologias assistivas como potencializador de inclusão de alunos com TEA (Outcome).

- Pontes et al. (2018) em seu trabalho identificam potencialidades e comparações entre as tecnologias digitais e recursos físicos na abordagem de crianças com TEA (Population); realizaram análise comparativa de recursos físicos como o Picture Exchange Communication System (PECS), Livro de velcro, troca de figuras e rotina diária e os aplicativos Livox®, Aboard®, Tobii Sono Flex ${ }^{\circledR}$, Autismo Imagem ${ }^{\circledR}$ e Discussão ${ }^{\circledR}$ (Intervention); Ambos os recursos podem mediar processos de ensino. Destacam que os aplicativos proporcionam novos caminhos para o desenvolvimento da aprendizagem e do cuidado integral (Outcome).

- Silveira et al. (2020) avaliaram aplicativos gratuitos para dispositivos móveis que promovam acessibilidade e inclusão digital e educacional para pessoas com TEA na Modalidade EAD (Population); Avaliaram aplicativos móveis como o

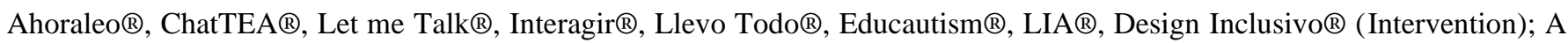
pesquisa verificou que os vários programas atendem às necessidades de pessoas com TEA. Se tais recursos forem incorporados a ambientes virtuais de aprendizado propiciariam inclusão de estudantes com TEA (Outcome).

- Soares et al. (2017) aplicaram um cenário de realidade aumentada em 4 crianças com TEA (Population); no estudo os autores relataram a produção de um ambiente de realidade aumentada e aplicação através do Google Card Board® para transformar a face humana em uma caricatura animada (Intervention); os resultados sugerem que a ferramenta é útil para auxiliar educadores e profissionais da saúde como apoio psicopedagógico de crianças com TEA. Pois os autores argumentam que esse projeto facilita a interação face a face de crianças com TEA com seus professores/cuidadores. (Outcome).

- Teixeira et al. (2017) estudaram o desenvolvimento das habilidades em crianças com o TEA (Population). Realizaram uma revisão da literatura que salientou a ferramenta Jclic ${ }^{\circledR}$ em crianças com TEA e a aplicaram em seu grupo de estudo (Intervention); os resultados mostram potencialidades do recurso tecnológico enquanto tecnologia assistida e de apoio didático para crianças com TEA em diferentes realidades sociais (Outcome).

Sobre os periódicos que publicaram tais artigos avaliados nesta pesquisa, aproximadamente 35,7\% ( $\mathrm{n}=5$ ) eram da área da interdisciplinar; cerca de 14,2\% ( $\mathrm{n}=2)$ eram da área de psicologia/educação; os demais trabalhos pertenciam a periódicos das áreas de ciências humanas, Educação, Ensino, Design/Tecnologia, ciências da computação e enfermagem.

Em relação ao ano de publicação, observou-se que aproximadamente $85,7 \%$ dos artigos foram publicados em cada ano de 2017 (n=4), 2018 (n=4) e 2020 (n=4); sendo uma publicação no ano de 2013 e de 2019, conforme Figura 1. 
Research, Society and Development, v. 10, n. 10, e119101018328, 2021

(CC BY 4.0) | ISSN 2525-3409 | DOI: http://dx.doi.org/10.33448/rsd-v10i10.18328

Figura 1. Distribuição das publicações por ano.

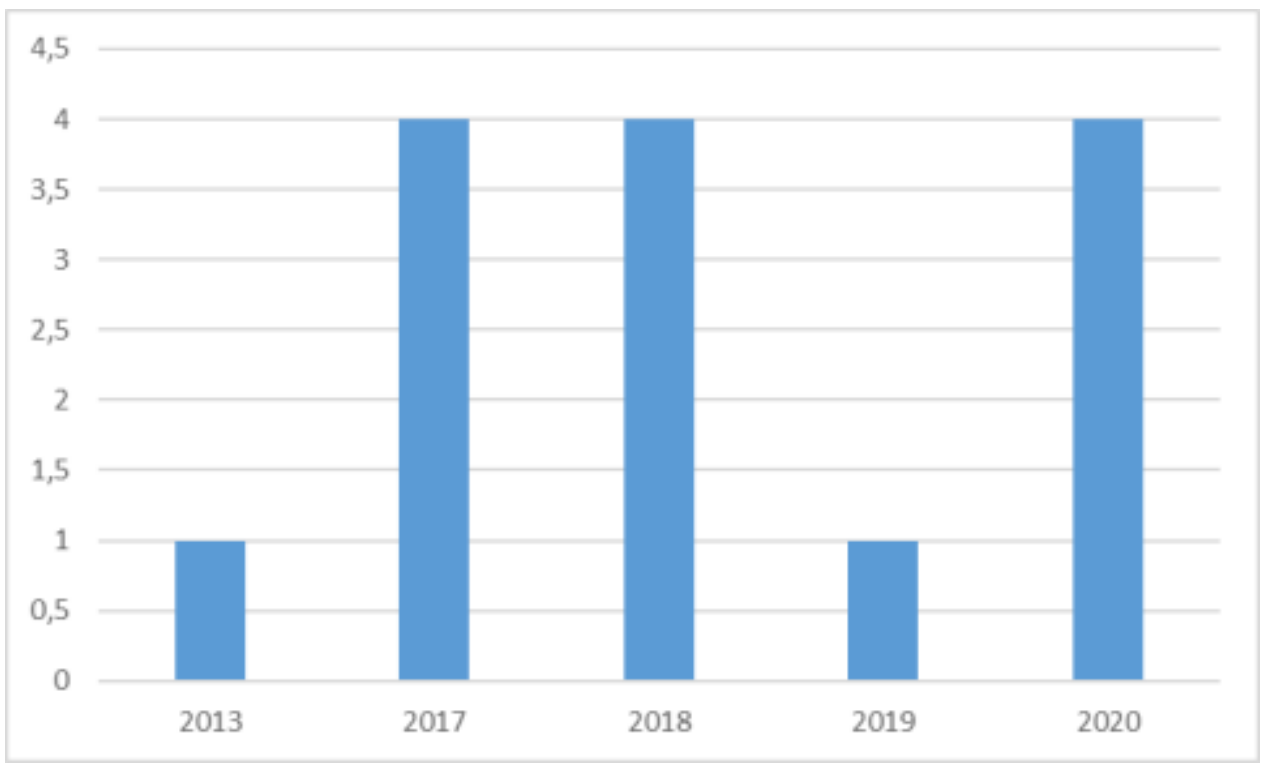

Fonte: Autores.

De todos os artigos selecionados, cerca de 21,4\% (n=3) são publicações internacionais, sendo duas mexicanas e 1 espanhola realizadas por pesquisadores brasileiros. O Estado do Rio Grande do Sul apresentou 35,7\% (n=5) das publicações, seguidos pelo Estado de São Paulo com 14,2\% (n=2); Paraná, Santa Catarina, Alagoas e Rio de Janeiro figuraram com cerca de $7,1 \%(n=1)$ para cada.

Verificou-se que aproximadamente 28,5\% (n=4) dos artigos selecionados e analisados desenvolveram algum aplicativo, interface personalizável, programa de realidade aumentada ou aplicativo de comunicação de metas para auxiliar o processo de ensino-aprendizagem de crianças com TEA; cerca de 35,7\% analisaram as tecnologias educacionais através de levantamento bibliográfico; próximo de 21,4\% aplicaram alguma tecnologia já existente na avaliação de desenvolvimento de crianças com TEA; e, aproximadamente, 14,2\% $(\mathrm{n}=2)$ realizaram estudos comparativos entre as tecnologias digitais e não digitais (meios físicos) aplicáveis aos pacientes com o TEA.

Apresenta-se também uma classificação do foco dos estudos quanto sua relação com as TIC’s, na Tabela 2 e na Figura 2. 
Tabela 2. Estudos analisados conforme classificação quanto ao foco da pesquisa em relação as TIC's.

\begin{tabular}{cc}
\hline Estudo analisado & Classificação quanto ao foco em relação as TIC's \\
\hline Passerino et al. (2013) & Análise de Software \\
\hline Andrade e Teodoro (2017) & Desenvolvimento de Software \\
\hline Brito e Novôa (2017) & Análise de Software \\
\hline Soares et al. (2017) & Desenvolvimento de Software \\
\hline Teixeira et al. $\mathbf{( 2 0 1 7 ) .}$ & Análise de Software \\
\hline Andreis e Rigo (2018) & Desenvolvimento de Software \\
\hline Oliveira et al. (2018) & Desenvolvimento de Software \\
\hline Pontes et al. (2018) & Análise de Software \\
\hline Kanashiro e Seabra júnior (2018) & Análise de Software \\
\hline Lucian e Stumpf (2019) & Análise de Software \\
\hline Maia e Jacomelli, (2020) & Levantamento Bibliográfico \\
\hline Nascimento et al. (2020) & Levantamento Bibliográfico \\
\hline De Bettio e Giacomazzo (2020) & Levantamento Bibliográfico \\
\hline Silveira et al. $\mathbf{( 2 0 2 0 )}$ & Análise de Software
\end{tabular}

Fonte: Autores.

Figura 2. Gráfico sobre os estudos analisados conforme classificação quanto ao foco da pesquisa em relação as TIC's.

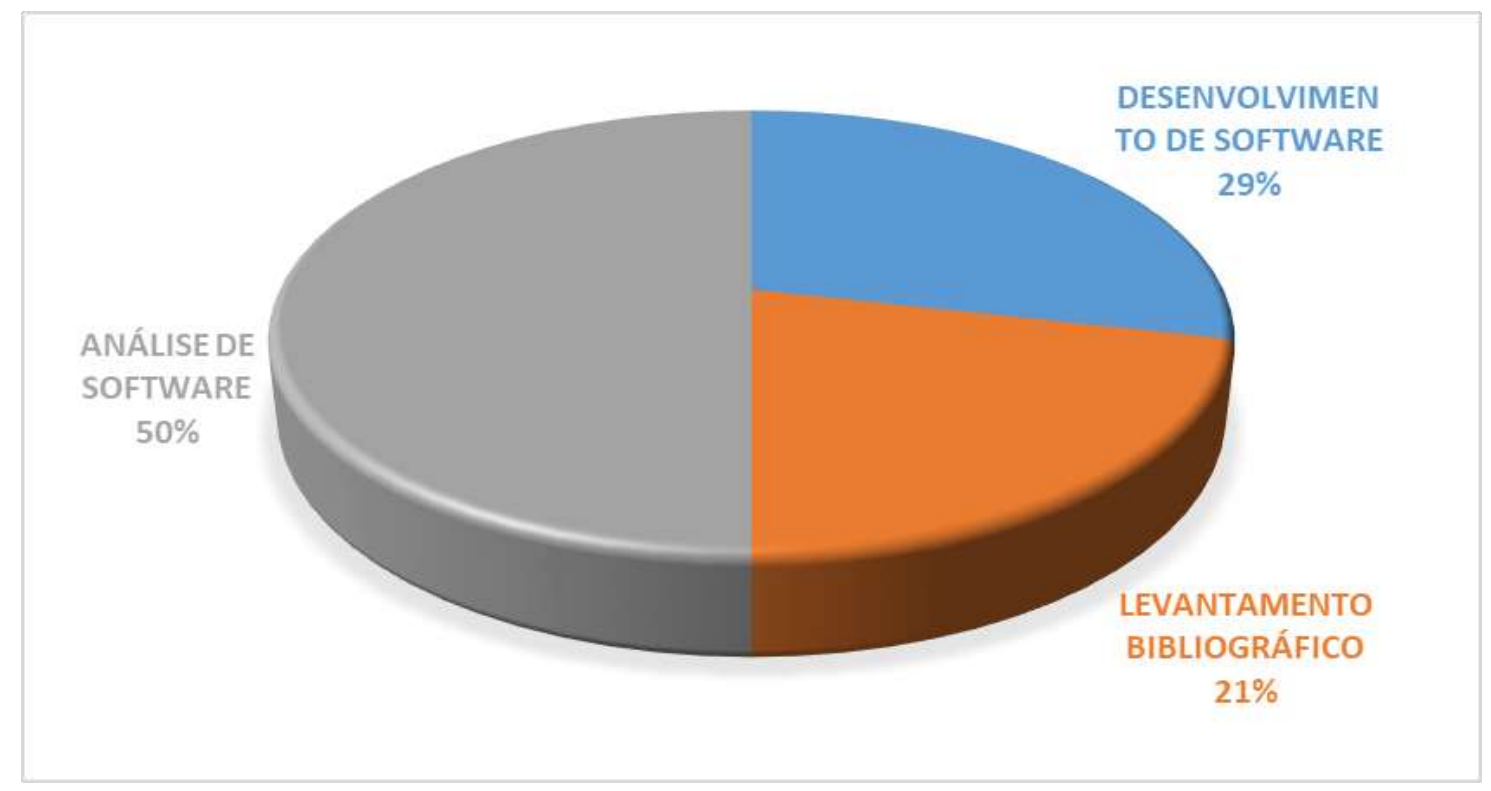

Fonte: Autores.

Os artigos avaliados nesta pesquisa, salientam uma potencial melhora no processo de educação, de interação interpessoal, da relação paciente com profissionais de saúde quando há utilização de TA e TIC's pelas crianças com o TEA.

\section{Discussão}

Nos modelos tradicionais de educação o ensino é baseado na comunicação verbal, o que dificulta indivíduos com o TEA pela própria característica do transtorno. Desde o século passado existe uma preocupação em pesquisar diferentes formas de auxiliar a apoiar o desenvolvimento de crianças com o TEA (Andreis \& Rigo, 2018). Dessa forma, pela própria dificuldade de interação social dos indivíduos com o TEA, deve-se atentar para práticas pedagógicas que trabalhem essa deficiência. Além do mais, as características do TEA representam um verdadeiro espectro no grau de comprometimento social e comportamento 
estereotipado, o que dificulta uma abordagem de rotina de forma a seguir protocolos, sendo uma abordagem complexa e, quase, individualizada, devendo os educadores estarem preparados para esta demanda.

O processo de aprendizagem dos alunos com o TEA é demorado, necessita de auxílio de um mediador para oferecer condições e instrumentos adequados para cada aprendente de acordo com suas especificidades (De Bettio \& Giacomazzo, 2020). É necessário mitigar a situação da criança com o TEA, desenvolver recursos, metodologias e tecnologias que possibilitem superar os déficits que acometem a pessoa, com intuito de melhorar as relações interpessoais, ampliar os processos de socialização para intensificar o desenvolvimento e a aprendizagem das pessoas com o TEA.

Ao realizar tal pesquisa, os autores acreditam que deveria existir um melhor preparo dos profissionais da educação para lecionarem de forma adequada para uma população com necessidades especiais como os indivíduos com o TEA, assim como uma melhor instrumentalização das escolas para que se tornem, efetivamente, inclusivas; pois para De Bettio \& Giacomazzo (2020), trabalhar com aprendizagem de crianças com o TEA não é igual ao trabalho com outros alunos, pois cada indivíduo dentro do espectro do transtorno é um universo com habilidades, comportamentos e personalidades diferentes.

Para Lucian e Stumpf (2019), dentro da amplitude do TEA, existem diversas dificuldades como na comunicação e relacionamento social, porém existem autistas com altas habilidades, com total independência, de modo a se revelarem como talentos específicos. De Bettio e Giacomazzo (2020) ressaltam que a aprendizagem não está inteiramente ligada ao cognitivo, mas sim a outras habilidades simples a serem realizadas sozinhas no dia-a-dia.

A educação de qualidade para os pacientes com necessidades especiais é garantida por lei, como consta no artigo 205 de Constituição Federal do Brasil. Existe também Lei Federal número 12.764, 27 de dezembro de 2012, que considera o TEA uma deficiência com todas as prerrogativas legais das pessoas com deficiência, preconiza a inserção no ensino regular crianças e adolescentes com o TEA. Dessa forma, pode-se dizer, de maneira simplista, que a capacitação profissional dos professores quanto à educação especial é obrigada por Lei, garantindo a inclusão.

Para Lucian e Stumpf (2019), a convivência na inclusão escolar propicia o desenvolvimento não só para o aluno com necessidades especiais, mas também para os demais colegas e funcionários, pelo fato de terem a oportunidade de conviverem e aprenderem com as diferenças.

$\mathrm{O}$ foco das pesquisas brasileira, analisadas neste trabalho, foi a revisão da literatura de forma a analisar as tecnologias já existentes. Contudo, cabe salientar que quase um terço dos trabalhos analisados desenvolveram uma tecnologia digital para crianças com TEA, fenômeno que foi identificado por Silveira et al (2020) que relatou o crescimento de produtos, tecnologias de software e aplicativos educacionais destinados para autistas, o que colabora com a atual motivação de inclusão efetiva desse público no cenário educacional, incluindo também a modalidade de ensino à distância. As crianças com o TEA, em suas mais variadas formas de aprender, precisam vivenciar diferentes possibilidades de aprendizagem com recursos tecnológicos, que poderão superar as barreiras e obstáculos impostos pela homogenização do ensino (Brito \& Novôa, 2017).

Deve-se aproveitar o interesse que uma criança com o TEA apresenta por máquinas fotográficas, computadores, celulares entre outros objetos, para estimular a sua interação com o interlocutor e o mundo em que vive (Maia \& Jacomelli, 2020).

Lucian e Stumpf (2019) salientam que os recursos digitais educacionais destinados às crianças com o TEA devam explorar o visual, as atividades interativas, os recursos de som, as imagens e animações para facilitar o aprendizado. Os autores afirmam ainda que os dispositivos móveis são capazes de exercer grande atratividade sobre indivíduos com o TEA, podendo auxiliar no desenvolvimento do aprendizado nas crianças com esse transtorno.

Nascimento et al. (2020) relatam em sua pesquisa a importância do suporte visual para introduzir novos conhecimentos quanto para o desenvolvimento de atividades para crianças com o TEA. Nesse sentido, é necessário que a sequência didática e materiais sejam planejados e desenvolvidos considerando as características específicas do aluno às que se 
destinam, devendo-se sempre pensar se o recurso é adequado ao aluno, pois estes possuem características muito diversificadas.

As tecnologias assistidas podem ser definidas como quaisquer recursos, digitais ou não, que promovam uma independência a indivíduos com alguma deficiência, conferindo a este, a possibilidade de melhorar sua vida diária.

Atualmente é possível encontrar diversos aplicativos voltados às pessoas com TEA, em especial as crianças. Estes aplicativos focam nas atividades específicas, enquanto há outros que visam trabalhar múltiplas habilidades em diferentes contextos escolar, doméstico, terapêutico entre outros (Lucian \& Stumpf, 2019).

Para De Bettio e Jacomazzo (2020) as tecnologias são essenciais na educação de crianças com o TEA, pois os alunos conseguem aperfeiçoar a linguagem, comunicação, expressam a oralidade e aprimoram o raciocínio lógico.

Kanashiro e Seabra Júnior (2018) demonstraram, através de seu experimento com o software denominado Jornada nas Estrelas, o desenvolvimento da aquisição da escrita em uma criança com o TEA que estava desnivelada a seus pares em uma escola com inclusão.

Brito e Novôa (2017) apresentam em seu trabalho algumas experiências de interação de tecnologias como recursos na ação pedagógica na educação especial. Analisaram recursos digitais como os softwares Amplisoft $®$, AIELLO®, Descobrindo Emoções ${ }^{\circledR}$, Lina Educa®, Scala®, Emoplay ${ }^{\circledR}$ e os aplicativos CAN Game Maker® e ABC Autismo®, além de tecnologias não digitais como painel de rotina. Frente as características do TEA, os autores destacam os softwares e aplicativos como ferramentas pedagógicas para o desenvolvimento da aprendizagem desses alunos.

Contudo, as tecnologias por si só não são capazes de desenvolverem melhoras no processo de ensino-aprendizagem de crianças com o TEA. Para Kanashiro e Seabra Júnior (2018), é relevante a ação didática do docente junto a tecnologia digital, uma vez que, sem orientação, direcionamento e incitação do professor não se conseguirá atingir o sucesso desejado. Nesse contexto, Maia e Jacomelli (2020) e Brito e Novôa (2017) afirmam que a tecnologia em si não oferece grandes aquisições de habilidades, necessitando de um intermediador: o professor/docente. Os autores frisam também que deve existir uma troca de estímulo entre professor e aluno e coleguinhas, estimulando-o a pensar e interagir e que o docente deve estimular o aluno respeitando suas especificidades, transpondo suas dificuldades.

Maia e Jacomelli (2020) realizaram um levantamento bibliográfico com o objetivo de identificar se as tecnologias da informação e comunicação (TIC) colaboram para a educação de crianças com o TEA. Concluem que o uso de aplicativos direcionados à aprendizagem interfere positivamente na formação da criança com o TEA como desenvolvendo autoestima, criatividade, pensamento lógico, coordenação motora, além de favorecer a aquisição de novos conhecimentos.

Passerino et al. (2013) apresentaram em seu artigo o projeto SCALA®, que é um sistema de comunicação para letramento de crianças com o TEA em salas multifuncionais. O sistema possibilitou criar pranchas de comunicação, narrativa, estratégia de letramento através do acesso em computadores ou tablets android em um cadastro gratuito.

Andrade e Teodoro (2017) desenvolveram, com auxílio de psicólogos e engenheiros, um aplicativo de comunicação de metas para os indivíduos com TEA denominado STEPP® (Sistema de Transparência de Equipe entre pais e profissionais). Os autores consideram que protótipo pode ter seu uso ampliado para diversas populações que requeiram tratamento multidisciplinar e estimulação intensiva.

O estudo de Soares et al. (2017) apresentou uma ferramenta de realidade aumentada para ajudar nas tarefas psicopedagógicas de crianças com o TEA. Os autores introduzem a ideia de que as perdas executivas nas crianças com o TEA são decorrentes do processamento facial que esta faz de seus professores/profissionais de saúde. Para embasar essa hipótese, os autores construíram um ambiente de realidade aumentada, utilizando tecnologia simples como os óculos de realidade virtual do Google Cardboard $\circledast$, que transforma a imagem capturada pela câmera de um celular em um desenho animado, similar a um gibi. Seus resultados evidenciam que a suavização da face humana com auxílio da tecnologia é relevante para melhor entendimento de atividades que envolvam as funções executivas, melhorando também a interação social dessas crianças. 
O trabalho de Teixeira et al. (2017) avalia o uso da ferramenta Jclic® para desenvolver as habilidades das crianças com o TEA. Os resultados demostraram as potencialidades desse recurso enquanto tecnologia assistida e de apoio didático para crianças com o TEA. Os autores descrevem o JClic ${ }^{\circledR}$ como um software educativo multimídia baseado em jogos e em padrões abertos para a utilização em qualquer sistema operacional. É composto por uma variedade de atividades educativas para ser utilizadas dentre ou fora da escola.

Andreis e Rigo (2018) apresentam um sistema desenvolvido com o intuito de resolver o problema de uso e adaptação à software de apoio à educação de crianças com o TEA. O Sistema Educautism ${ }^{\circledR}$ apresenta uma nova proposta de interface personalizável de acordo com o perfil da criança. Os resultados mostraram que mesmo ainda não sendo um aplicativo completo, o sistema proposto pode auxiliar na resolução na educação de crianças com TEA.

O trabalho de Oliveira et al. (2018) objetivou desenvolver e aplicar um sistema de Web inovador, em que apresenta de modo digital as atividades de ensino-aprendizagem para crianças com TEA utilizando a metodologia TEACCH (Treament and Education of Autistic and related Communication Handi-capped Children), que se apresentou de forma eficiente com melhora nos resultados cognitivos das crianças avaliadas pós aplicação do sistema.

Existem alguns métodos educacionais para minimizar as incapacidades geradas pelo TEA. A metodologia TEACCH advoga que o autismo pode ser melhor compreendido como um transtorno invasivo do desenvolvimento humano e que a criança com esse problema evoluirá com um tratamento que associe a terapia comportamental e educação especial, assim sendo, trata-se de um método psicoeducacional que assegura que as crianças com o TEA responderão positivamente às realidades estruturadas de acordo com as suas limitações e potencialidades (Teixeira et al., 2017).

No trabalho de Lucian e Stumpf (2019) foram estudados e analisados 3 aplicativos utilizáveis pelos indivíduos com o TEA, que são o ABC autismo®, TEO Autismo ${ }^{\circledR}$ e Autismo Projeto Integrar ${ }^{\circledR}$. Os autores elencaram o ABC® autismo para realizar os testes de usabilidade em crianças com TEA, em que constataram interação satisfatória e envolvimento da criança com o aplicativo.

Pontes et al. (2020) avaliaram e compararam os aplicativos digitais e os recursos físicos para ajudar na comunicação de crianças com o TEA. Concluem que ambos recursos podem mediar o processo de comunicação de crianças com o TEA e destacam que os aplicativos contribuem para aprimorar a qualidade das interações sociais nestas crianças.

Silveira et al. (2020), no contexto da educação a distância (EAD), elencam aplicativos gratuitos em dispositivos móveis como o Ahoraleo ${ }^{\circledR}$, ChatTEA $®$, Letmetalk, Interagir ${ }^{\circledR}$, LLevotodo $®$, Educautism $®$, LIA $®$ e Design Inclusivo para autistas ${ }^{\circledR}$. Os autores consideram que os aplicativos contribuem, enquanto tecnologias assistidas, para inclusão digital dos indivíduos com o TEA na modalidade EAD.

Além das tecnologias digitais mais estudadas como aplicativos ou softwares, os desenhos animados e filmes infantis podem ser considerados métodos educativos tecnológicos. Para Maia e Jacomelli (2020), a programação infantil na televisão proporciona uma aprendizagem qualitativa, desde que com a mediação do professor questionando a personagem, suas ações, o enredo e entre outras ações pedagógicas.

Os referidos autores elencam desenhos animados que auxiliam o desenvolvimento de crianças com o TEA, são eles: "Dora, a aventureira", "O show da Luna", "Princesa Sofia", "Léo, o guarda da floresta", "Patrulha canina”, "As aventuras de Tainá”.

Além desses desenhos citados, pode-se referenciar, seguindo a mesma linha de benefícios para a criança com o TEA que assista à programação, o desenho infantil "Caillou", pode-se mostrar benéfico para instrumentalização da criança e, com isso, ajudar e seu desenvolvimento holísitco, desde que mediada pelos educadores. O seriado de desenho infantil mostra um menino de 4 anos descobrindo o mundo em enredos como a família, sociedade, comportamento social, amizades e também sobre as emoções. A série é encontrada no Brasil nos canais Discovery Kids, TV Cultura, além de youtube e netflix. Outro 
desenho animando que se pode destacar com o mesmo propósito é o "Daniel Tigre" que narra o cotidiano das crianças préescolares. O desenho transmite diversos ensinamentos quanto a presença da família, a escola, os vizinhos. O protagonista, Daniel, chama o telespectador de vizinho, mantendo um diálogo como se a criança que o assiste também participasse no enredo. No Brasil, a série é exibida pelo Disney Junior e pela TV Brasil, além de estar disponível no youtube.

Tais desenhos e programas infantis apresentam personagens caracterizados, faces agradáveis e simpáticas, o que pode atrair a atenção das crianças com o TEA. Para Soares et al. (2017) evidenciam que a hipótese por eles levantada procede nas crianças com o TEA. Os autores desenvolveram um ambiente composto por um aplicativo que caricaturiza a face humana, em tempo real, através dos frames capturados pela câmera de um smartphone e é suavizado através de óculos de realidade virtual que suportam a plataforma androide. A suavização facial através da tecnologia, proposta no artigo, demonstra uma relevante melhora das atividades das funções executadas pelas crianças com TEA.

A utilização de recursos audiovisuais para a formação de uma criança com o TEA ultrapassa o entretenimento, pois quando assistidos com ênfase educativa estimulam a linguagem, compreensão do enredo e há transposição de conhecimento, além de possibilitar o surgimento se emoções, quase inexistente nas crianças com o TEA (Maia \& Jacomelli, 2020).

A crescente importância tecnológica no cotidiano nas últimas décadas atinge a educação especial, em uma tentativa de facilitar o processo de ensino-aprendizagem de alunos com algum transtorno, como o TEA. Percebe-se que $21 \%$ dos estudos incluídos neste artigo são de levantamento bibliográfico, enquanto que $79 \%$ tratam diretamente de TIC's, sendo 50\% de análise de software e $29 \%$ de desenvolvimento de software. Adicionalmente, em parte devido à mudança de nomenclatura de Autismo para "Transtorno do Espectro Autista", verifica-se um maior número de publicações nos anos de 2017, 2018 e 2020.

Os artigos avaliados neste mapeamento sistemático apresentam, em sua maioria, uma proposta interdisciplinar. Tal cenário pode ser percebido pela variedade de periódicos que foram identificados em diversas áreas da saúde, informática e educação. Sendo assim, as melhorias na qualidade de vida ou no processo de ensino-aprendizagem deve ser multidisciplinar, ou melhor, translacional, com um arcabouço de ideias das diversas áreas do conhecimento de saúde, educacional e de tecnologias.

\section{Conclusão}

Os trabalhos avaliados neste Estudo descrevem revisões da literatura e experimentos de comparação de tecnologias em diversos regiões e estados do Brasil, além de desenvolvimento de tecnologias digitais, o que deve ser salientado por ser uma atividade inovadora. Em sua maioria foram publicados em revistas da área interdisciplinar, o que evidencia a necessidade de inter-relação entre as áreas do conhecimento que se dedicam à essa população em especial.

Frente a pesquisa realizada, a inserção das TIC's nas tecnologias assistivas pode-se tornar uma intervenção útil em indivíduos com TEA, melhorando seus processos de comunicação e até de cognição. O processo ensino-aprendizagem já é uma difícil tarefa, sendo mais complexa nas crianças com o TEA, pois se depara com uma variedade de pré-requisitos como adequação de escolas, empoderamento dos professores no que tange as tecnologias digitais, além de acompanhamento translacional multiprofissional com identificação precisa dos déficits de cada criança para poder trabalhá-las no ambiente escolar com uma determinada tecnologia, ou seja, um diagnóstico holístico do quadro clínico-educacional da criança.

Os artigos analisados não determinam ou apontam para uma melhor metodologia a ser aplicada, apenas fazem referências como promissores os métodos e tecnologias já existentes ou criados pelos próprios autores, sendo necessário planejamento didático, mais conhecimentos dos professores sobre as tecnologias e aplicação adequada de cada tecnologia à necessidade de cada aprendente com TEA. Sendo assim, é difícil prescrever uma tecnologia para uma intervenção educacional em um paciente específico sem uma prévia avaliação completa, realizada por uma equipe de especialistas. No entanto, em relação às pesquisas analisadas, observou-se que $79 \%$ dos estudos tratam diretamente de TIC's, sendo 50\% de análise de 
software e $29 \%$ de desenvolvimento de software, o que mostra uma tendência nas pesquisas brasileiras nos últimos 4 anos, de 2017 a 2020. Isto é, o interesse da comunidade científica brasileira está crescendo quanto a aplicação e desenvolvimento de novas tecnologias para TEA.

\section{Referências}

American Psychiatric Association (2013). Manual de diagnóstico e estatístico de transtornos mentais: DSM-V(5a ed.). Artmed.

Andrade, A. A. \& Teodoro, M. L. M. (2017). Desenvolvimento de um aplicativo de comunicação de metas entre cuidadores e profissionais de saúde no tratamento de pessoas com Transtorno do Espectro do Autismo. Revista Tecnologia e Sociedade, 13(27), 139-155. https://www.redalyc.org/pdf/4966/496654014010.pdf

Andreis, I. \& Rigo, S. J. (2018). EDUCAUTISM: Um sistema personalizável para o apoio à educação de crianças diagnosticadas com o transtorno do espectro autista. RENOTE-Revista Novas Tecnologias na Educação, 16(1). https://www.seer.ufrgs.br/renote/article/view/85924

Brasil. Constituição Federal de 1988. Artigo 208. https://www.senado.leg.br/atividade/const/con1988/con1988_18.02.2016/art_208_.asp\# : : :text=O\%20dever $\% 20$ do $\% 20$ Estado $\% 20$ com,efetivado $\% 20$ mediante $\% 20$ a $\% 20$ garantia $\% 20 \mathrm{de} \% 3 \mathrm{~A} \&$ text=VII\%20\%2D $\% 20$ atendimento $\% 20$ ao $\% 20$ educand o\%2C\%20em,alimenta\%C3\%A7\%C3\%A3o\%20e\%20assist\%C3\%AAncia\%20\%C3\%A0\%20sa\% 3\%BAde

Brito, G.S. \& Novôa, J. (2017). Tecnologias como Ferramentas de Ensino e Formação Continuada na Educação Especial. Revista Eletrônica Argentina-Brasil de Tecnologias da Informação e da Comunicação, 1(7). https://revistas.setrem.com.br/index.php/reabtic/article/view/215

De Bettio, T. \& Giacomazzo, G. F. (2020). A tecnologia assistiva e a aprendizagem dos alunos com transtorno do espectro autista: análise das pesquisas. Revista Saberes Pedagógicos, 4(1), 260-280. http://periodicos.unesc.net/pedag/article/view/5745

Kanashiro, M. D. D. M. \& Seabra Junior, M. O. (2018). Tecnologia educacional como recurso para a alfabetização da criança com transtorno do espectro autista. Revista Diálogos e Perspectivas Em Educação Especial, 5(2), 101-120. https://revistas.marilia.unesp.br/index.php/dialog oseperspectivas/article/view/8773

Lei $\mathrm{n}^{o}$ 12.764, de 27 de dezembro de 2012. (Institui a Política Nacional de Proteção dos Direitos da Pessoa com Transtorno do Espectro Autista). http://www.planalto.gov.br/ccivil_03/_ato20112014/2012/lei/112764.htm

Lucian, B. O. \& Stumpf, A. (2019). Análise de aplicativos destinados ao aprendizado de crianças com transtorno do espectro autista. Design e Tecnologia, 9(19), 43-65. https://www.ufrgs.br/det/index.php/det/article/view/601/281

Maia, M. S. D. \& Jacomelli, M. K. (2020). A Aprendizagem da Criança com Transtorno do Espectro Autista (TEA) através do Uso das Tecnologias da Informação e Comunicação-TIC. Revista Psicologia \& Saberes, 9(18), 16-31. https://revistas.cesmac.edu.br/index.php/psicologia/article/view/1210

Moreira, P. L., Silva Júnior, J. I. \& Silva, V. M. (2016). Um breve guia para Revisões Sistemáticas aplicado a Ciência da Computação. Palmas-TO: EDUFT.

Mukherjee, S. B., Kapoor, S. \& Sharma, S. (2021). Identification of Essential, Equivocal and Complex Autism by the Autism Dysmorphology Measure: An Observational Study. Journal of Autism and Developmental Disorders, 51(5), 1550-1561, https://doi.org/10.1007/s10803-020-04641-x

Nascimento, A. G. C., Luna, J. M., Esquincalha, A. C. \& Santos, R. G. C. (2020). Educação Matemática para estudantes autistas: conteúdos e recursos mais explorados na literatura de pesquisa. Boletim Gepem, 76, 63-78. http://costalima.ufrrj.br/index.php/gepem/article/view/605

Oliveira, M. A. C. S., Guebert, M. C. C., Nohama, P. (2018). TEACCH Methodology-Based Web System to Support Learning for Children with Autism. IEEE Latin America Transactions, 16(11), 2698-2705. https://ieeexplore.ieee.org/abstract/document/8795110

Passerino, L. M., Bez, M. R., Vicari, R. M. (2013). Formação de professores em comunicação alternativa para crianças com TEA: contextos em ação. Revista Educação Especial, 26(47), 619-638. https://periodicos.ufsm.br/index.php/educacaoespecial/article/view/10475

Pontes, E. L., Kleparde, A. A., Silva, T. P., Puggina, A. C. G., Apostolico, M. R. \& Pino-Oliveira, A. A. (2018). Tecnologias Digitais e Recursos Físicos Na Abordagem de Crianças Com Transtorno do Espectro Autista. Revista Saúde-UNG-Ser, 12 (3/4), 68-74. http://revistas.ung.br/index.php/saude/article/view/3729

Richardson, W. S., Wilson, M. C., Nishikawa, J. \& Hayward, R. S. (1995). The well-built clinical question: a key to evidence-based decisions. ACP J Club, 123(3), 12-3. https://www.acpjournals.org/doi/10.7326/ACPJC-1995-123-3-A12

Silveira, L. C. G., Luiz, J. M., Guterres, L. X., Mendes, L. F. S. \& Ribeiro, L. O. M. (2020). Tecnologias Assistivas no contexto da acessibilidade e mobilidade: possibilidades de inclusão digital de autistas na educação a distância. EmRede-Revista de Educação a Distância, 7(2), 61-73. https://www.aunirede.org.br/revista/index.php/emrede/article/view/539

Soares, K. P., Burlamarqui, A. M. F., Gonçalves, L. M. G., Costa, V. F., Cunha, M. E. \& Burlamarqui, A. A. R. S. S. (2017). Preliminary studies with augmented reality tool to help in psycho-pedagogical tasks with children belonging to autism spectrum disorder. IEEE Latin: America Transactions, 15(10), 2017-2023. https://ieeexplore.iee.org/abstract/document/8071250

Teixeira, M., Santos, A., Aquino, C. \& Medeiros, E. (2017). Utilizando o software JCLIC enquanto tecnologia assistiva no tratamento do autismo. Revista de Estudios e Investigación en Psicología y Educación, Extr.(13). https://core.ac.uk/download/pdf/229588704.pdf 